\title{
fLPS 2.0: rapid annotation of compositionally-biased regions in biological sequences
}

\author{
Paul M Harrison ${ }^{\text {Corresp. } 1}$ \\ ${ }^{1}$ Dept. of Biology, McGill University, Montreal, QC, Canada \\ Corresponding Author: Paul M Harrison \\ Email address: paul.harrison@mcgill.ca
}

Compositionally-biased $(\mathrm{CB})$ regions in biological sequences are enriched for a subset of sequence residue types. These can be shorter regions with a concentrated bias (i.e., those termed 'low-complexity'), or longer regions that have a compositional skew. These regions comprise a prominent class of the uncharacterized 'dark matter' of the protein universe. Here, I report the latest version of the fLPS package for the annotation of CB regions, which includes added consideration of DNA sequences, to label the eight possible biased regions of DNA. In this version, the user is now able to restrict analysis to a specified subset of residue types, and also to filter for previously annotated domains to enable detection of discontinuous CB regions. A 'thorough' option has been added which enables the labelling of subtler biases, typically made from a skew for several residue types. In the output, protein CB regions are now labelled with bias classes reflecting the physicochemical character of the biasing residues. The fLPS 2.0 package is available from: https://github.com/pmharrison/flps2 or in a supplementary file of this paper. 
2 fLPS 2.0: rapid annotation of compositionally-biased regions

3 in biological sequences

4

5 Paul M. Harrison

6 Department of Biology,

7 McGill University,

8 Montreal, QC,

9 Canada.

10

11 Corresponding author:

12 Paul Harrison

13 Email: paul.harrison@mcgill.ca

14

15

16 
17 Abstract: Compositionally-biased (CB) regions in biological sequences are enriched

18 for a subset of sequence residue types. These can be shorter regions with a concentrated

19 bias (i.e., those termed 'low-complexity'), or longer regions that have a compositional

20 skew. These regions comprise a prominent class of the uncharacterized 'dark matter' of

21 the protein universe. Here, I report the latest version of the fLPS package for the

22 annotation of $\mathrm{CB}$ regions, which includes added consideration of DNA sequences, to label

23 the eight possible biased regions of DNA. In this version, the user is now able to restrict

24 analysis to a specified subset of residue types, and also to filter for previously annotated

25 domains to enable detection of discontinuous CB regions. A 'thorough' option has been

26 added which enables the labelling of subtler biases, typically made from a skew for

27 several residue types. In the output, protein CB regions are now labelled with bias classes

28 reflecting the physico-chemical character of the biasing residues. The fLPS 2.0 package

29 is available from: https://github.com/pmharrison/flps2 or in a supplementary file of this 30 paper.

\section{Introduction}

33 Biological sequences, despite being made from fixed alphabets of residues,

34 demonstrate a wide diversity of sequence compositions. In particular, these sequences

35 can be compositionally biased (CB) for a subset of the residue alphabet. For example,

36 the protein sequence tract EDEEKDDELEIEEDEEDDDEDEDED is biased for $\mathrm{E}$ and $\mathrm{D}$

37 (glutamate and aspartate). If these tracts are sufficiently biased or repetitive over a short

38 stretch, then they are termed 'low-complexity'. Also, one can have longer tracts that

39 exhibit a milder compositional skew. In between, there are a continuum of CB cases 
40 (Harrison 2006; Harrison \& Gerstein 2003). In proteins, CB regions are linked to distinct

41 biophysical states such as intrinsic disorder, and to cell-structural proteins, fibrous

42 proteins, and functional amyloids and prions (Harbi \& Harrison 2014; Harrison 2006), and

43 to the formation of intracellular biomolecular condensates or membraneless organelles

44 (Gomes \& Shorter 2019). They also comprise part of the protein 'dark matter' that remains

45 largely un- or under-characterized (Harrison 2018); indeed, some CB dark matter is not 46 assignable as intrinsically disordered or structured, and may give us clues to as yet 47 unknown biophysical protein states (Harrison 2018).

48 Several programs to annotate CB regions-and in particular, low-complexity (LC) regions—have been developed. These include SIMPLE (Hancock \& Armstrong 1994), 50 SEG (Wootton \& Federhen 1996), CAST (Promponas et al. 2000), 0j.py (Wise 2001), 51 ScanCom (Nandi et al. 2003), CARD (Shin \& Kim 2005), BIAS (Kuznetsov \& Hwang 2006), LCD-Composer (Cascarina et al. 2021) and LPS / fLPS (Harrison 2006; Harrison 2017; Harrison \& Gerstein 2003). SEG annotates LC sequences by performing a scan using thresholds for sequence entropy and a fixed window length. It is used for masking LC sequences as part of the BLAST sequence alignment package (Altschul et al. 1997). Such masking is sometimes needed to avoid false inference of similarity by evolutionary descent (since these simpler sequences can arise independently multiple times during evolution quite easily). CAST annotates LC sequence by aligning to homopeptides of the twenty amino acids (Promponas et al. 2000). LCD-Composer uses a measure of amino-

60 acid dispersion to characterise low complexity (Cascarina et al. 2021). The LCT web 61 server analyzes the low-complexity and 'repeatability' of proteins sequences with a 62 graphical output (Mier \& Andrade-Navarro 2020). Two other servers LCReXXXplorer and 
63 PLaToLoCo combine the results of multiple programs to graphically display LC regions 64 (Jarnot et al. 2020; Kirmitzoglou \& Promponas 2015). The LPS algorithm used binomial

65 probability to check for low-probability sequence regions, and was further developed into 66 the fast algorithm fLPS, which can annotate the TrEMBL database in $<1$ hour (Harbi et 67 al. 2011; Harrison 2017; Harrison \& Gerstein 2003). This algorithm has been applied successfully to the analysis of prions and prion-like proteins, and protein 'dark matter' (An et al. 2016; An \& Harrison 2016; Harbi et al. 2011; Harrison et al. 2007; Harrison 2018; Harrison 2020; Su \& Harrison 2019; Su \& Harrison 2020).

The fLPS program is especially useful for analyzing $C B$ regions since: (i) it analyzes the full range of $\mathrm{CB}$ types (from low complexity to milder compositional skews); (ii) it characterizes both single- and multiple-residue biases; (iii) it does not require the specification of residue types by the user (although this option has now been added); (iv) it considers the differing background frequencies of individual residue types; $(v)$ it is faster than the commonly used SEG algorithm (Harrison 2017). fLPS has been applied to, for example, the identification of transactivation domains \{Arnold, $2018 \# 33$, to analysis of the conservation of low-complexity regions in prokaryotes \{Ntountoumi, $2019 \# 34$ \}, analysis of low-complexity regions in stress granules $\{$ Zhu, $2020 \# 35\}$, and the delineation 80 of domains in kinetochore proteins \{Cortes-Silva, $2020 \# 36\}$ and in the PRR19 protein

81 that functions in meiotic crossing over \{Bondarieva, $2020 \# 37$, as well as in studies of

82 prion-like protein evolution \{Harrison, 2020 \#26\}\{An, 2016 \#3;An, 2016 \#2\}\{Su, 2019 $83 \# 39 ;$ Su, $2020 \# 38$. Here, the latest fLPS 2.0 package is reported. In this package, the 84 program flow has been modified to add consideration of DNA sequences; also, the user 85 can specify subsets of residue types and existing domain annotations to filter from 
86 sequences, in order to discover discontinuous biased regions. The baseline precision of

87 the algorithm can be adjusted to discover more mildly biased regions that may have

88 biological significance. Examples of fLPS 2.0 application are presented and discussed.

89

90 Methods

91 Implementation

92 The fLPS 2.0 package is written in standard C. The name 'fLPS' stands for fast

93 LPS, where LPS stands for 'Low Probability Subsequences'. The package comprises the

94 source code and executables compiled for MacOSX and Linux. There is the fLPS

95 program itself, plus two accessory programs: CompositionMaker, which can be used to

96 calculate background residue compositions; and DomainFilter, which is used to either

97 excise or mask previously annotated domains (such as those with known protein

98 structure, see section immediately below). Each of the programs works on input files of

99 any size in standard FASTA format. The package is available at

100 https://github.com/pmharrison/flps2 and in Supplementary File 1.

102 Algorithm and new added features

103 The program fLPS works through a process of binomial probability (P-value)

104 minimization, as described in detail previously (Harrison 2017). There are four main steps

105 that are summarized in Figure 1 at the top of the figure: (i) QUICK SCAN; (ii) MINIMIZE;

106 (iii) MERGE; (iv) OUTPUT. At the end of the process, single-, and multiple-residue LPSs,

107 are output if they are below the user-specified P-value threshold, or default threshold.

108 Biased regions are labelled with a bias signature which is a list of the biasing residues in 
109 order of bias precedence delimited with curly brackets. At each of these stages, efficiency

110 measures are taken to avoid or delay probability calculations unless/until they are

111 necessary (Harrison 2017).

112 The following are the main new options added to the fLPS code:

113 (i) Precision of the calculation: In the initial QUICK SCAN step, by default (the ' $-z$

114 fast' option), windows with a P-value below the baseline threshold of 0.001 are

115 considered. Also, the windowing along the sequence proceeds with a step size

$116=3$ residues (Figure 1 ). This means that some regions that are made from

117 biases for a larger number of residue types might be missed; also, short regions

118 with a milder bias that might have biological significance could sometimes be

119 overlooked. Therefore, options for the base-line precision of the program have

120 been added. If ' $-z$ medium' is specified, the base-line P-value threshold is set

121 to 0.01 , with a windowing step size $=2$. For the most precise option $-z$

122 thorough', the base-line P-value is 0.1 and the step size $=1$ (Figure 1$)$.

123 However, these latter two options can produce a huge amount of output for

124 larger databases, so they should be applied to such databases with caution.

125 (ii) DNA analysis: DNA sequences can be specified using the $-n$ option. By default,

126 each of the four bases A, G, C and T has equal background probability.

127 (iii) Domain filtering: Using the DomainFilter accessory program, previously 128 annotated domains can be filtered in either of two ways, i.e., either 'excised' or 'masked'. When 'excised' is specified, DomainFilter outputs shorter sequences, with the domain sequences removed. The 'masked' option outputs sequences with the domains masked with Xs. The positions of the excised or masked 
domains are labelled on the name line of the sequences in the FASTA-format file. When the FASTA-format output file from DomainFilter is used as input for fLPS bias annotations, the domain positions appear in the fLPS output if the option $-D$ is specified.

(iv) Restriction lists: With the $-r$ option, the user can specify a subset of residue types, e.g., only negatively-charged amino acids (E, D), or the six-membered $\operatorname{aromatics}(\mathrm{F}, \mathrm{Y}, \mathrm{W})$.

140 output filename that also contains the parameters used in running the fLPS program; a

141 '-o oneline' output option, wherein the results for each sequence are listed in a single-

142 line summary; $a-k$ option to ignore the unknown residues in calculations (' $X$ ' for proteins

143 and ' $N$ ' for DNA). The output has also been updated to include further new features. A

144 calculation of the enrichment of the biasing residues in the output LPSs has been added,

145 which is the proportion of biasing residues in the LPS divided by the total expected

146 background frequencies of the biasing residues. To enable quicker characterization of

147 bias trends in a data set, 'bias class' labels are now featured for both protein and DNA

148 sequences. For proteins, these labels are derived from the Taylor amino-acid 149 classification Venn diagram, with some additional categories (Taylor 1986). The 150 applicable class label that has the smallest membership is picked, when assigning these.

151 For DNA, these labels represent the eight possible compositional biases: $\{A\}|\{T\},\{G\}|\{C\}$, $152\{A T\},\{G C\},\{A C\}|\{G T\},\{A G\}|\{C T\},\{A T C\} \mid\{A T G\}$ and $\{A C G\} \mid\{C G T\}$ (discussed below).

153 For better annotation of short low-complexity regions, trimming of LPSs of minimum 154 window length is now employed. That is, if possible, residues are sheared off both ends 
155 of the minimum-length LPS if they do not contribute to the bias. This improves the

156 annotation of $\sim 6-8 \%$ of LPSs, in trials on the S. cerevisiae S288C proteome (downloaded

157 from UniProt reference proteomes (Boeckmann et al. 2003)) using a variety of 158 parameters.

160 Example data

161 The UniProt canonical reference human and budding yeast (S. cerevisiae strain

162 288C) proteomes were downloaded from www.uniprot.org in January 2021 (Boeckmann

163 et al. 2003). The human proteome was cross-referenced with the InterPro list of domain

164 annotations downloaded from http://ebi.ac.uk/interpro, to make a list of human proteins

165 that contain the RRM RNA-binding domain (Blum et al. 2021).

166 Human promoter data was obtained from the EPD eukaryotic promoter database

167 (Schmid et al. 2004). These were a set of representative promoters (one per gene)

168 defined by the EPD. Sequences spanning from -999 to +100 around the transcription

169 start site were analysed.

170

171 Prion-like regions

172 Prion-like regions were annotated for the human proteome using the PLAAC 173 program with default parameters (Lancaster et al. 2014).

175 Results

176 Using the new domain-filtering and restriction list options of $f L P S$ : application to analysis

177 of human RNA-binding proteins 
It is often advantageous to restrict CB annotation to a subset of residues to enable

179 easy counting of different types of bias region. Users are now able to restrict their bias

180 annotation using a 'restriction list' specified with the $-r$ option of fLPS (Figure 2a). Also, it

181 is possible that certain proteins have discontinuous CB regions, i.e., the CB regions may

182 have small, structured domains embedded in them, or they may be comprised of the loop

183 regions within a single protein domain. To enable discovery of such discontinuous CB

184 regions, the DomainFilter program can be used to excise or mask domains or domain

185 parts before using the fLPS program (Figure 2a). These two options were combined in

186 analyzing the CB regions of human RNA-binding proteins, specifically those containing

187 the RRM RNA-binding domain (Figure 2). The RRM domain is used in eukaryotes to bind

188 RNA during diverse cellular processes, and is typically associated with intrinsically-

189 disordered regions (Su \& Harrison 2020). After applying DomainFilter to excise Pfam

190 protein domain annotations (Mistry et al. 2021), the main single-residue biases were

191 assessed with an initial run of fLPS (those having $>50$ cases); thereafter, a final run of

192 fLPS used a restriction list based on these main single-residue biases to enable better

193 counting of bias types.

194 There are 206 RRM-domain-containing human proteins in this protein data set.

195 The most common multiple- and single-residue biases involve arginine, serine, proline

196 and glycine, and are associated mostly with 'mixed', 'polar', 'small' and 'charged' bias

197 classes; glutamine and asparagine biases, which are associated typically with prion-like

198 domains, are only of middling abundance (Figure 2b-d). Indeed, although prion-like

199 domains are often cited as being associated with RNA-binding proteins, in this case they

200 only occur in $\sim 1$ in 6 RRM-containing proteins, as judged by the PLAAC program 
201 (Lancaster et al. 2014) (Suppl. Figure 1, 32/206 (15.5\%) have PLAAC LLR scores $\geq 15.0$,

202 and 35/206 (17.0\%) have PLAAC PRD scores >15.0). These PLAAC prion-like regions

203 arise despite only moderate asparagine and glutamine frequencies and are thus

204 substantially dependent on other residues that are common in prion-forming domains,

205 such as tyrosine, glycine and serine, which are common biases in the RRM-containing

206 proteins (Figure 2b-d). In Figure 2e, the human BOLL 'protein boule-like' is presented as

207 an example of a discontinuous CB domain around an RRM domain.

208

209 Increased precision with the $-\mathrm{z}$ option

210 As described in Methods, the $-z$ option can be used to increase the precision of

211 the initial scanning by the fLPS algorithm for compositional deviations (Figure 1). Two

212 examples of the effects of this option are illustrated (Figure 3). Multi-protein bridging factor

213 MBF1 is a transcriptional coactivator that promotes GCN4-dependent transcriptional

214 activity by bridging between the DNA-binding areas of GCN4 and TATA-binding protein.

215 The default fLPS settings detect a mild bias for positively-charged residues $\{K R\}$, which

216 becomes a stronger bias comprised of further biasing residues $\{K R Q G A N V S P\}$ when the

217 'thorough' option is applied. Also, a region weakly biased for polar residues $\{T D N\}$

218 appears (Figure 3a). These biases are likely linked to DNA and protein interactions within

219 complexes. The second example is the Shadoo protein from human (Figure 3b). Shadoo

220 is a member of the prion-protein ( $\operatorname{PrP})$ family that has demonstrated some neuroprotective

221 behaviour (Westaway et al. 2011). Like PrP, the protein that underlies prion diseases, it

222 contains $\mathrm{CB}$ and intrinsically-disordered regions. Here, the major $\mathrm{CB}$ annotations are

223 stable when $-z$ thorough is specified, but additional mildly biased regions are detected, 
224 one of which corresponds to a signal peptide, the other an area bridging between and

225 intrinsically disordered region and a pro-peptide (Figure 3b).

226 These examples demonstrate three effects of increasing the precision of the initial

227 compositional scanning: (i) mildly biased tracts are detected that can be quite short and

228 that may have biological significance; (ii) further bias detail is sometimes added to CB

229 regions, decreasing the binomial P-value; (iii) tracts with a bias made from several

230 residues and that were previously not detectable (such as the $\{$ AWLC $\}$ tract in Figure 1(b))

231 become evident. In aggregate, these three effects increase the ability of the program to

232 delineate compositionally-defined domains in proteins. As shown in Table 1 detailing

233 analysis of the $S$. cerevisiae proteome, a significant number of further multiple-residue

234 CB regions are detected, even for smaller $\mathrm{P}$-value thresholds (such as $\mathrm{P} \leq 1 \mathrm{e}-09$ ). Since

235 one of the tracts in Figure 1(b) corresponds to a signal peptide, the correspondence

236 between signal peptide positions in the S. cerevisiae proteome and CB regions was also

237 examined (Table 2). The number of signal peptides corresponding to $\mathrm{CB}$ regions

238 increases to a highest value of $\sim 60 \%$ with a $\mathrm{P} \leq 1 \mathrm{e}-03$ bias $\mathrm{P}$-value threshold. The results

239 are generally in line with a previous analysis of sequence complexity in signal peptides,

240 where $24 \%$ of residues of signal peptides in analyzed data sets were labelled part of low-

241 complexity tracts by SEG $\{$ Wong, 2010 \#42\}\{Wootton, $1996 \# 5\}$.

242

243 Analysis of DNA sequence

244 DNA sequences can be analyzed by specifying the $-n$ option; by default, each

245 base is expected with equal background probability. In total, there are 40 different

246 possible biases (Figure 4a). These can be segregated into eight bias classes for DNA 
247 (Figure 4a). The last two of these bias classes, $\{A T C\} \mid\{A T G\}$ and $\{A C G\} \mid\{C G T\}$,

248 correspond to strand-specific depletions of single bases, i.e., $\{$ ATC\}|\{ATG\} indicates a

249 strand-specific lack of $\mathrm{C}$ or $\mathrm{G}$. An example of a DNA CB region from a human promoter

250 is illustrated (Figure 4b). To illustrate its application to DNA, we used the fLPS program

251 to examine bias trends in a representative data set of human promoters taken from the

252 EPD database (Figure 4c). Interestingly, the tri-base $\{A T C\} \mid\{A T G\}$ and $\{A C G\} \mid\{C G T\}$ bias

253 classes are almost as prevalent as the two-base $\{A C\} \mid\{G T\}$ bias class.

254

255 Discussion

256

The known protein universe contains much 'dark matter', some structured, some

257 intrinsically disordered, some not assignable as either (Harrison 2018). The present fLPS

258 package helps to address one aspect of this protein dark matter, which is that it often has

259 unusual amino-acid composition, the structural properties of which have yet to be

260 characterized. As examined above for human RRM-domain-containing proteins, some of

261 this dark matter may be discontinuous CB regions that have smaller structured domains

262 embedded in them. Such discontinuity might also be possible within a protein domain,

263 e.g., in the loops of a transmembrane domain. fLPS can also be used to assess whether

264 such compositional biases are unusual relative to the background proteome composition

265 of a particular organism or clade, or are part of organism- / clade-specific trend. CB

266 domains, such as the proline-rich region in PRR19 protein \{Bondarieva, $2020 \# 37$ \} that

267 functions in meiotic crossing-over, may have specific functional relevance. Parsing

268 proteins into subdomains, including milder CB domains found with an increased baseline

269 thoroughness (the $-z$ option), may help in the generation of experimental constructs and 
270 further hypotheses for experiments. They are also useful for studying proteome-wide

271 trends to gain more general functional or evolutionary insights (e.g., refs. \{Ntountoumi,

$2722019 \# 34\}\{\mathrm{Su}, 2020 \# 38\}$ ). Varying the parameters (in particular $-m,-M,-t$, and the new

273 parameters $-r$ and $-z$ ) can help to delineate possible biologically meaningful subdomains

274 in larger biased tracts or within intrinsically disordered regions, such as in prion-forming

275 proteins $\{$ Harrison, 2017 \#22\}.

276

The fLPS package program flow was modified to accommodate the option of

277 analyzing DNA sequences. I applied this option to a set of representative human

278 promoters, as an example. Beyond the standard conception of DNA bias as either \{GC\}

279 or $\{A T\}$, substantial tracts of other possible biases were observed, including strand-

280 specific dearths of single bases (i.e., the bias classes $\{A T C\} \mid\{A T G\}$ and $\{A C G\} \mid\{C G T\}$ ). It

281 would be interesting to investigate experimentally whether such DNA CB domains have

282 a general biological significance. To my knowledge, there is not a currently available

283 program that delineates all of the possible biased domains of DNA in this way (other

284 programs, such as Dustmasker \{Morgulis, $2006 \# 40\}$ or TANTAN \{Frith, $2011 \# 41\}$, are

285 designed to tackle the problem of avoiding spurious alignments, which is not what fLPS

286 is designed for.)

287 Intrinsically disordered regions (IDRs) in proteins were initially discovered as long

288 stretches of amino acids in proteins that remain unfolded under physiological conditions

289 [1, 2]\{Uversky, 2002 \#28\}. Compositional bias or 'low complexity' is a characteristic

290 feature of intrinsically disordered regions (IDRs), although there is substantial overlap in

291 sequence complexity values between IDRs and ordered regions \{Pedro Romero, 2001

$292 \# 27$ \}. Also, different definitions of sequence complexity or compositional bias have 
293 different degrees of linkage to disorder or order, with tandem-repeat tracts more likely to

294 encode ordered regions \{Mier, 2020 \#29\}. Because of this link, fLPS 2.0 may be useful

295 for the characterization of subdomains in intrinsically-disordered proteins. The boundaries

296 of compositionally-defined domains may differ to those of IDRs, IDRs may be split into multiple

297 compositionally-defined regions, or new algorithmic scenarios using the definition of

298 compositionally defined domains may enable the annotation of further intrinsic disorder $\{\mathrm{Necci}$,

$2992021 \# 30\}\{$ Sirota, $2010 \# 31\}\{$ Tang, $2021 \# 32\}$.

300

301 Examples of running the package

302 A diverse choice of parameters is possible in running the fLPS 2.0 program. Here 303 are some examples:

304 (1) Annotating low-complexity regions: For the specific task of annotating short CB 305 regions of the sort termed 'low-complexity', the following parameters are suitable 306 (with the yeast proteome 'yeast.fasta' as an example input file):

$$
\text { .fLPS -t1e-5 (or-t1e-6) -m5 -M25 -o long yeast.fasta }
$$

(2) Analyzing for discontinuous $C B$ domains: Firstly, structured domains are excised from the sequences, then fLPS is run using the -D option: $. / f L P S-D$ yeast.Dexcised.fasta 

.fFLS - dv -ooneline - c yeast.fasta.COMPOSITION -r FYW yeast.fasta Also specified are headers and footers $(-d)$, oneline output format (-o oneline) and verbose behaviour during runtime $(-v)$.

(4) Annotating longer biased regions with the thorough option: To find longer biased

Here, headers and footers are also output (-d option).

Conclusions

The fLPS 2.0 package is a versatile package for annotating compositional biases, either 'low-complexity' regions, or regions with milder or long-range compositional skew. Users can now apply the package to DNA to identify all the possible DNA CB domains. In addition to the unique features of fLPS listed at the end of the Introduction, utility is gained from the added domain filtering, restriction list and precision options, which can

332 be combined to identify CB domains in support of experimental hypotheses. The package 333 is available from: https://github.com/pmharrison/flps2 or Supplementary File 1.

Figure 1: A schematic detailing the $-z$ option to adjust the base-line precision of

338 the fLPS calculation. At the top of the figure is a pipeline summarizing the basic fLPS

339 algorithm. Below that is detailed the effect of the $-z$ option for adjusting the base-line 
340 precision of the algorithm. In the QUICK SCAN stage, when the $-z$ thorough option is

341 specified, more windows are stored in accord with the higher base-line P-value (these are

342 coloured green). Thus, there are more and longer search contigs (surrounded with yellow

343 box) at the end of this stage.

344

345 Figure 2: Analysis of RRM RNA-binding domain proteins in the human proteome.

346 (a) Human Pfam domain annotations (coloured boxes) are excised with DomainFilter and

347 the biases are annotated using fLPS 2.0 with human proteome background composition.

348 The most prevalent single-residue biases (occurring $>50$ times) were picked out (listed in

349 part (c)) and used as a restriction list with the $-r$ option. (b) A bar chart of the most

350 prevalent multiple-residue bias signatures (that occur for any threshold $\geq 3$ times). The

351 data for four P-value thresholds are shown. (c) As in (b) except that single-residue biases

352 are counted. (d) As in (b), except the bias classes are counted. The following bias classes

353 do not occur: glx, tiny_polar, polar_aromatic, aliphatic, aromatic. (e) An example of a

354 discontinuous biased region from human BOLL protein. The RRM domain (Pfam

355 PF00076, underlined bold) is excised. A $\{P\} C B$ region with $\mathrm{P}$-value $=7.2 \mathrm{e}-9$ is shown

356 in italics with the $P$ residues in red. There are also a $\{Y\} C B$ region $(P=8.6 e-6$, residues

357 in green) and a $\{Q\} C B$ region $(P=2.8 e-5$, residues in blue $)$. These go together to make

358 a $\{P Y Q\}$ region of the same extent as the $\{P\}$ region with $P=4.4 \mathrm{e}-13$. Other $Q$ and $Y$

359 residues in this multiple-residue CB region are in bold.

360

361 Figure 3: Two examples of the effect of the $-z$ option: (a) Multiprotein-bridging factor

362 MBF1 from S. cerevisiae; (b) human Shadoo protein. 
363 Single-residue CB regions are depicted as blue boxes and multiple-residue as green.

364 They are labelled with their biases and binomial P-values, and their endpoints. Intrinsic

365 disorder and other domain annotations are labelled in orange and grey respectively (and

366 are taken from UniProt (Boeckmann et al. 2003)). At the top of each panel are depicted

367 the annotation from the default $-z$ fast option, below that the annotations after using the

$368-z$ thorough option, then at the bottom of each panel are the UniProt sequence 369 annotations.

371 Figure 4: Analysis of DNA: (a) Eight classes of bias are possible in DNA.

372 Complementary biases are arrayed above and below the line, i.e., a bias on one strand

373 for $\{G T\}$ (guanine and thymidine) corresponds to a bias for $\{C A\}$ (cytidine and adenine)

374 on the complementary strand. Biases with the same colour are summarized with one of

375 the eight basic bias class labels (in the box at the bottom of the panel).

376 (b) An example of a biased region in human promoter DNA, for colipase CLPS_1.

377 The position in the promoter (downloaded from the EPD database (Schmid et al. 2004))

378 is indicated, along with the bias signature $\{A C\}$.

379 (c) Prevalences of the eight bias classes in human promoters downloaded from 380 the EPD (Schmid et al. 2004). Data for each of three bias P-value thresholds are shown 381 ( $\mathrm{P} \leq 1.0 \mathrm{e}-06, \mathrm{P} \leq 1.0 \mathrm{e}-09$ and $\mathrm{P} \leq 1.0 \mathrm{e}-12)$. The total number of residues in $\mathrm{CB}$ regions for

382 each of these thresholds is summed and depicted in natural logarithmic scale. The 383 numeric values are labelled on the top of each bar. 
386 Altschul SF, Madden TL, Schaffer AA, Zhang J, Zhang Z, Miller W, and Lipman DJ. 1997. Gapped BLAST and PSI-BLAST: a new generation of protein database search programs. Nucleic Acids Res 25:3389-3402.

An L, Fitzpatrick D, and Harrison PM. 2016. Emergence and evolution of yeast prion and prion-like proteins. BMC Evol Biol 16:24. 10.1186/s12862-016-0594-3

An L, and Harrison PM. 2016. The evolutionary scope and neurological disease linkage of yeast-prion-like proteins in humans. Biol Direct 11:32. 10.1186/s13062-0160134-5

Blum M, Chang HY, Chuguransky S, Grego T, Kandasaamy S, Mitchell A, Nuka G, Paysan-Lafosse T, Qureshi M, Raj S, Richardson L, Salazar GA, Williams L, Bork P, Bridge A, Gough J, Haft DH, Letunic I, Marchler-Bauer A, Mi H, Natale DA, Necci M, Orengo CA, Pandurangan AP, Rivoire C, Sigrist CJA, Sillitoe I, Thanki N, Thomas PD, Tosatto SCE, Wu CH, Bateman A, and Finn RD. 2021. The InterPro protein families and domains database: 20 years on. Nucleic Acids Res 49:D344D354. 10.1093/nar/gkaa977

Boeckmann B, Bairoch A, Apweiler R, Blatter MC, Estreicher A, Gasteiger E, Martin MJ, Michoud K, O'Donovan C, Phan I, Pilbout S, and Schneider M. 2003. The SWISSPROT protein knowledgebase and its supplement TrEMBL in 2003. Nucleic Acids Res 31:365-370.

Cascarina SM, King DC, Osborne Nishimura E, and Ross ED. 2021. LCD-Composer: an intuitive, composition-centric method enabling the identification and detailed functional mapping of low-complexity domains. NAR Genom Bioinform 3:Iqab048. 10.1093/nargab/lqab048 
409 Gomes E, and Shorter J. 2019. The molecular language of membraneless organelles. J

$410 \quad$ Biol Chem 294:7115-7127. 10.1074/jbc.TM118.001192

411 Hancock JM, and Armstrong JS. 1994. SIMPLE34: an improved and enhanced 412 implementation for VAX and Sun computers of the SIMPLE algorithm for analysis 413 of clustered repetitive motifs in nucleotide sequences. Comput Appl Biosci 10:6770. $10.1093 /$ bioinformatics/10.1.67

415 Harbi D, and Harrison PM. 2014. Classifying prion and prion-like phenomena. Prion 8:pii: 41627960.

417 Harbi D, Kumar M, and Harrison PM. 2011. LPS-annotate: complete annotation of compositionally biased regions in the protein knowledgebase. Database (Oxford) 2011:baq031. 10.1093/database/baq031

Harrison LB, Yu Z, Stajich JE, Dietrich FS, and Harrison PM. 2007. Evolution of budding 421 yeast prion-determinant sequences across diverse fungi. J Mol Biol 368:273-282.

Harrison PM. 2006. Exhaustive assignment of compositional bias reveals universally prevalent biased regions: analysis of functional associations in human and Drosophila. BMC Bioinformatics 7:441. 10.1186/1471-2105-7-441

Harrison PM. 2017. fLPS: Fast discovery of compositional biases for the protein universe. BMC Bioinformatics 18:476. 10.1186/s12859-017-1906-3

Harrison PM. 2018. Compositionally Biased Dark Matter in the Protein Universe. Proteomics 18:e1800069. 10.1002/pmic.201800069

430 Harrison PM. 2020. Variable absorption of mutational trends by prion-forming domains during Saccharomycetes evolution. PeerJ 8:e9669. 10.7717/peerj.9669 
432 Harrison PM, and Gerstein M. 2003. A method to assess compositional bias in biological 433 sequences and its application to prion-like glutamine/asparagine-rich domains in 434 eukaryotic proteomes. Genome Biol 4:R40. 10.1186/gb-2003-4-6-r40

435 Jarnot $\mathrm{P}$, Ziemska-Legiecka J, Dobson L, Merski M, Mier P, Andrade-Navarro MA, 436 Hancock JM, Dosztanyi Z, Paladin L, Necci M, Piovesan D, Tosatto SCE, 437 Promponas VJ, Grynberg M, and Gruca A. 2020. PlaToLoCo: the first web meta-

Kirmitzoglou I, and Promponas VJ. 2015. LCR-eXXXplorer: a web platform to search, server for visualization and annotation of low complexity regions in proteins.

Kuznetsov IB, and Hwang S. 2006. A novel sensitive method for the detection of userdefined compositional bias in biological sequences. Bioinformatics 22:1055-1063. Nucleic Acids Res 48:W77-W84. 10.1093/nar/gkaa339 visualize and share data for low complexity regions in protein sequences. Bioinformatics 31:2208-2210. 10.1093/bioinformatics/btv115

Mier P, and Andrade-Navarro MA. 2020. Assessing the low complexity of protein sequences via the low complexity triangle. PLOS ONE 15:e0239154. 10.1371/journal.pone.0239154

Mistry J, Chuguransky S, Williams L, Qureshi M, Salazar GA, Sonnhammer ELL, Tosatto 453 SCE, Paladin L, Raj S, Richardson LJ, Finn RD, and Bateman A. 2021. Pfam: The 
454

455

456

457

458

460

461

462

463

464

465

466

467

468

469

470

471

472

473

474

475

476

protein families database in 2021. Nucleic Acids Res 49:D412-D419. 10.1093/nar/gkaa913

Nandi T, Dash D, Ghai R, C BR, Kannan K, Brahmachari SK, Ramakrishnan C, and Ramachandran S. 2003. A novel complexity measure for comparative analysis of protein sequences from complete genomes. J Biomol Struct Dyn 20:657-668. 10.1080/07391102.2003.10506882

Promponas VJ, Enright AJ, Tsoka S, Kreil DP, Leroy C, Hamodrakas S, Sander C, and Ouzounis CA. 2000. CAST: an iterative algorithm for the complexity analysis of sequence tracts. Complexity analysis of sequence tracts. Bioinformatics 16:915922.

Schmid CD, Praz V, Delorenzi M, Perier R, and Bucher P. 2004. The Eukaryotic Promoter Database EPD: the impact of in silico primer extension. Nucleic Acids Res 32:D8285. $10.1093 /$ nar/gkh122

Shin SW, and Kim SM. 2005. A new algorithm for detecting low-complexity regions in protein sequences. Bioinformatics 21:160-170. 10.1093/bioinformatics/bth497

Su TY, and Harrison PM. 2019. Conservation of Prion-Like Composition and Sequence in Prion-Formers and Prion-Like Proteins of Saccharomyces cerevisiae. Front Mol Biosci 6:54. 10.3389/fmolb.2019.00054

Su WC, and Harrison PM. 2020. Deep conservation of prion-like composition in the eukaryotic prion-former Pub1/Tia1 family and its relatives. PeerJ 8:e9023. 10.7717/peerj.9023

Taylor WR. 1986. The classification of amino acid conservation. J Theor Biol 119:205218. $10.1016 / \mathrm{s} 0022-5193(86) 80075-3$ 
477 Westaway D, Daude N, Wohlgemuth S, and Harrison P. 2011. The PrP-like proteins 478 Shadoo and Doppel. Top Curr Chem 305:225-256. 10.1007/128_2011_190

479 Wise MJ. 2001. 0j.py: a software tool for low complexity proteins and protein domains. 480 Bioinformatics 17 Suppl 1:S288-295. 10.1093/bioinformatics/17.suppl_1.s288

481 Wootton JC, and Federhen S. 1996. Analysis of compositionally biased regions in 482 sequence databases. Methods Enzymol 266:554-571.

483 
Figure 1

A schematic detailing the $-z$ option to adjust the base-line precision of the fLPS calculation.

At the top of the figure is a pipeline summarizing the basic fLPS algorithm. Below that is detailed the effect of the $-z$ option for adjusting the base-line precision of the algorithm. In the QUICK SCAN stage, when the $-z$ thorough option is specified, more windows are stored in accord with the higher base-line P-value (these are coloured green). Thus, there are more and longer search contigs (surrounded with yellow box) at the end of this stage.

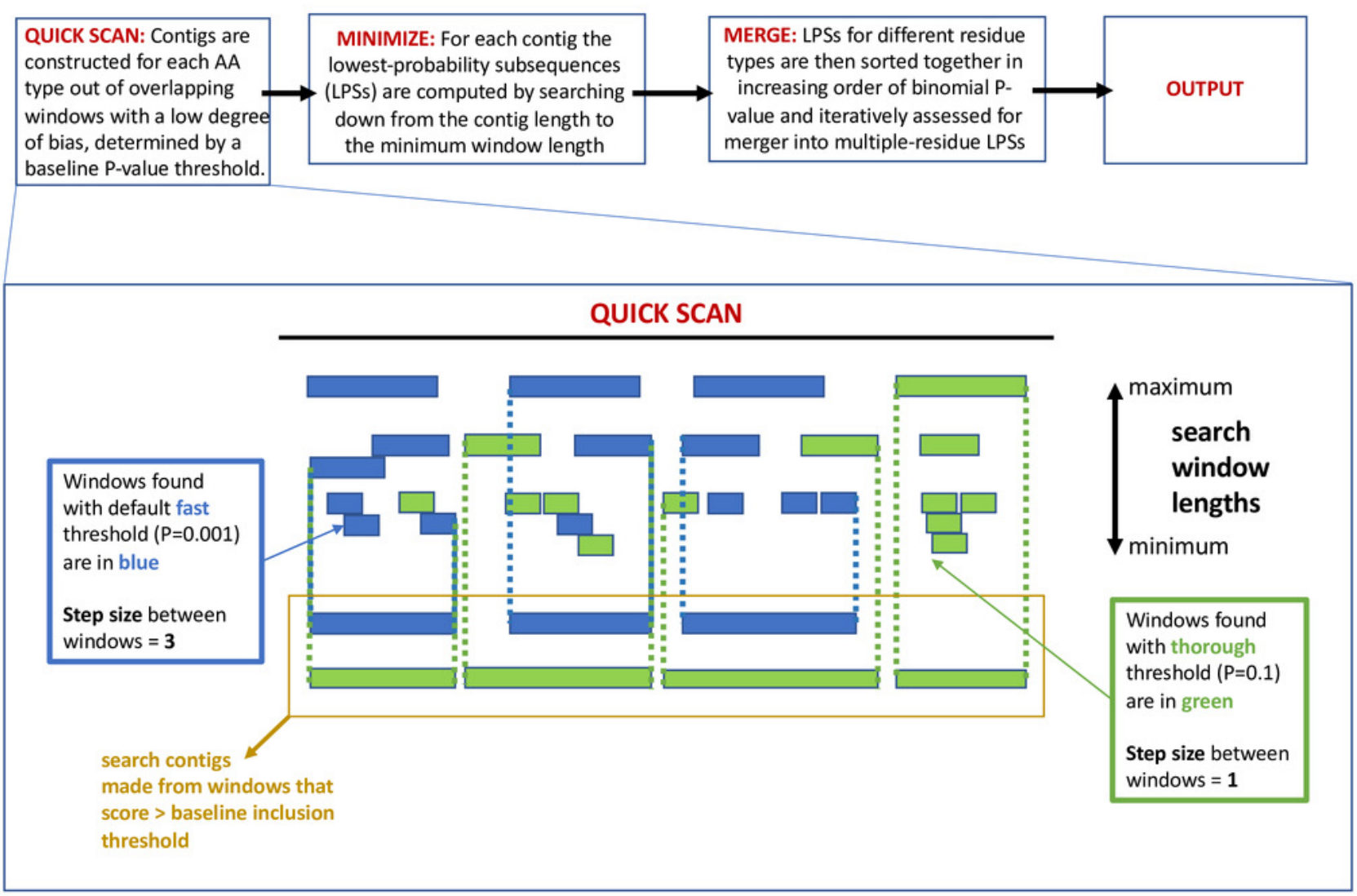




\section{Figure 2}

Analysis of RRM RNA-binding domain proteins in the human proteome.

(a) Human Pfam domain annotations (coloured boxes) are excised with DomainFilter and the biases are annotated using fLPS 2.0 with human proteome background composition. The most prevalent single-residue biases (occurring $>50$ times) were picked out (listed in part (c)) and used as a restriction list with the -r option. (b) A bar chart of the most prevalent multiple-residue bias signatures (that occur for any threshold $\geq 3$ times). The data for four Pvalue thresholds are shown. (c) As in (b) except that single-residue biases are counted. (d) As in (b), except the bias classes are counted. The following bias classes do not occur: glx, tiny_polar, polar_aromatic, aliphatic, aromatic. (e) An example of a discontinuous biased region from human BOLL protein. The RRM domain (Pfam PF00076, underlined bold) is excised. $A\{P\} C B$ region with $\mathrm{P}$-value $=7.2 \mathrm{e}-9$ is shown in italics with the $\mathrm{P}$ residues in red. There are also a $\{Y\} C B$ region $(P=8.6 e-6$, residues in green) and a $\{Q\} C B$ region $(P=$ $2.8 \mathrm{e}-5$, residues in blue). These go together to make a $\{P Y Q\}$ region of the same extent as the $\{P\}$ region with $P=4.4 \mathrm{e}-13$. Other $\mathrm{Q}$ and $\mathrm{Y}$ residues in this multiple-residue $\mathrm{CB}$ region are in bold. 
(a)

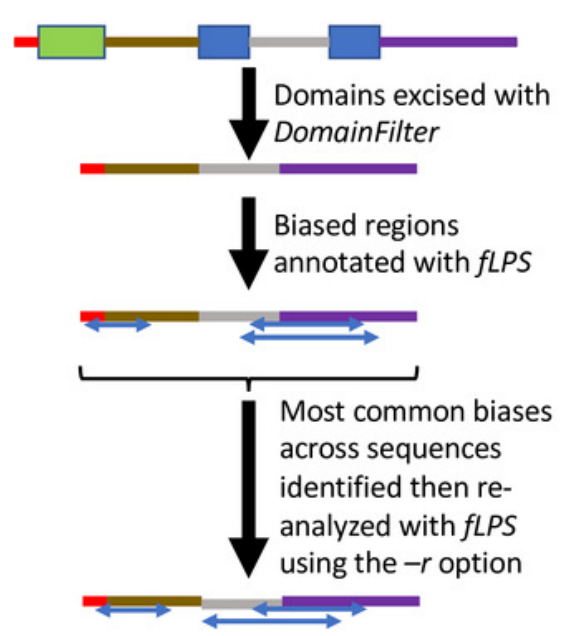

(e)

>Protein boule-like (BOLL) Q8N9W6

MQTDSLSPSPNPVSPVPLNNPTSAPRYGTVIPNR IFVGGIDFKTNESDLRKFFSQYGSVKEVKIVNDR AGVSKGYGFVTFETQEDAOKILQEAEKLNYKDKK LNIGPAIRKQQVGIPRSSIMPAAGTMYLTTSTGY PYTYHNGVAYFHTPEVTSVPPPWPSRSVCSSPVM $V A Q P I Y Q Q P A Y H Y Q A T T Q Y L P G Q W Q W S V P Q P S A S$ SAPFL YLOPSEVIYOPVEIAODGGCVPPPLSLME TSVPEPYSDHGVQATYHQVYAPSAITMPAPVMQP EPIKTVWSIHY (b)



(d) 100

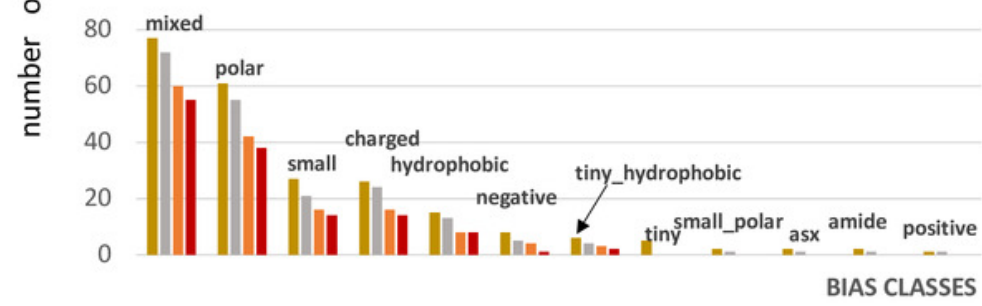




\section{Figure 3}

Two examples of the effect of the $-z$ option: (a) Multiprotein-bridging factor MBF1 from S. cerevisiae; (b) human Shadoo protein.

Single-residue CB regions are depicted as blue boxes and multiple-residue as green. They are labelled with their biases and binomial P-values, and their endpoints. Intrinsic disorder and other domain annotations are labelled in orange and grey respectively (and are taken from UniProt (Boeckmann et al. 2003) ). At the top of each panel are depicted the annotation from the default $-z$ fast option, below that the annotations after using the $-z$ thorough option, then at the bottom of each panel are the UniProt sequence annotations. 


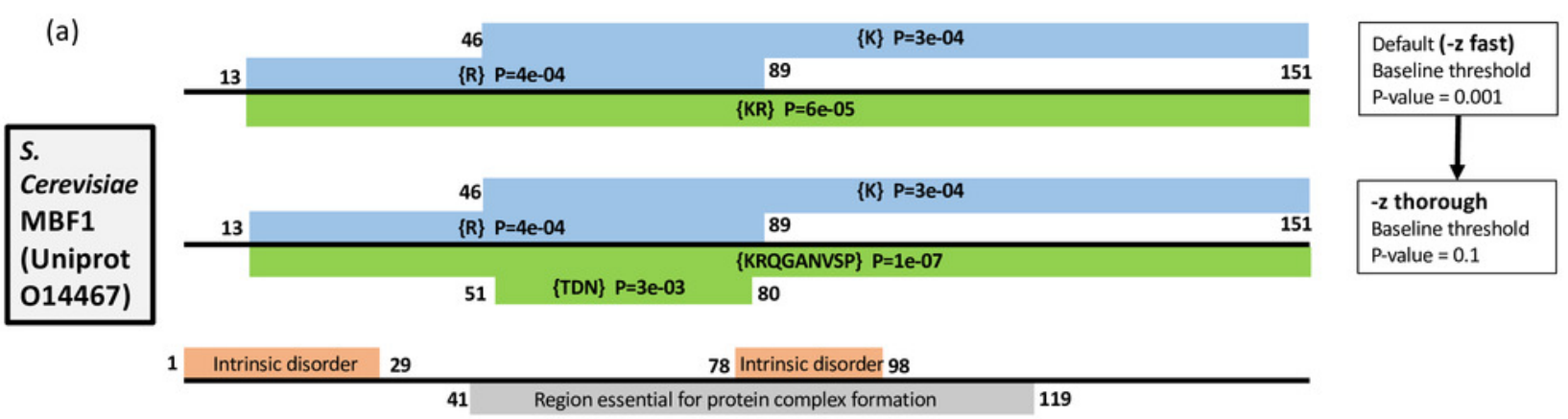

(b)

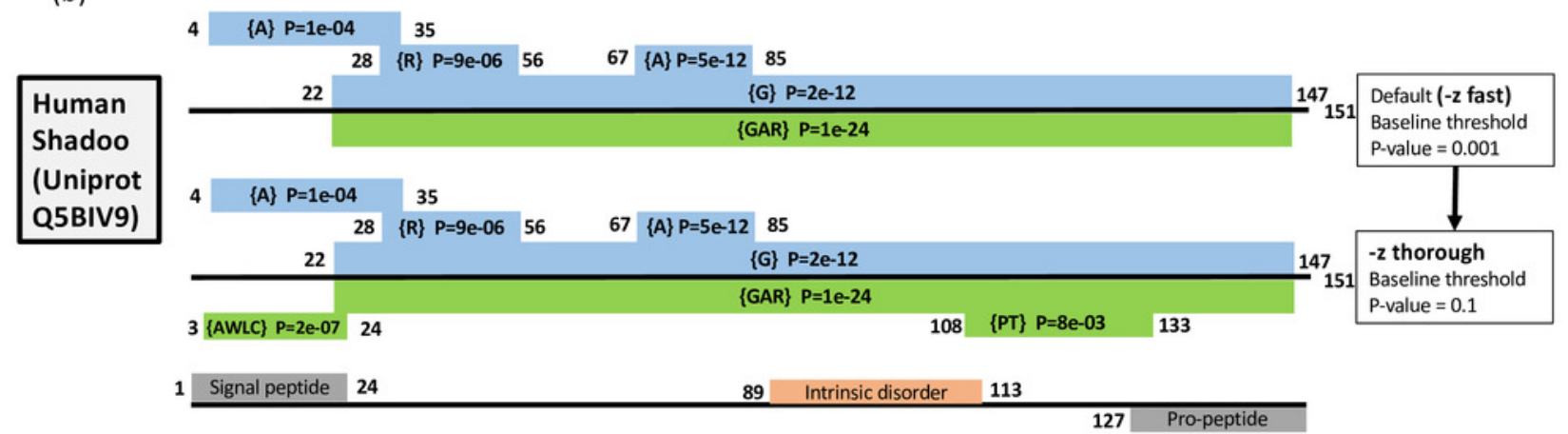




\section{Figure 4}

\section{Analysis of DNA}

(a) Eight classes of bias are possible in DNA. Complementary biases are arrayed above and below the line, i.e., a bias on one strand for $\{G T\}$ (guanine and thymidine) corresponds to a bias for $\{C A\}$ (cytidine and adenine) on the complementary strand. Biases with the same colour are summarized with one of the eight basic bias class labels (in the box at the bottom of the panel). (b) An example of a biased region in human promoter DNA, for colipase CLPS_1. The position in the promoter (downloaded from the EPD database (Schmid et al. 2004) ) is indicated, along with the bias signature $\{A C\}$. 
(a)

The eight classes of bias in DNA

$\{T\} \quad\{C\} \quad\{T A\} \quad\{C G\} \quad\{T G\}\{G T\}\{T C\}\{C T\}\{T A G\}\{T A C\}\{G T A\}\{G A T\}\{A G T\}\{A C T\}\{G A C\}\{G C A\}\{C A G\}\{C G A\}\{A G C\}\{A C G\}$

A $\}\{$ G $\} \quad\{A T\} \quad\{G C\}\{A C\}\{C A\}\{A G\}\{G A\}\{A T C\}\{A T G\}\{C A T\}\{C T A\}\{T C A\}\{T G A\}\{C T G\}\{C G T\}\{G T C\}\{G C T\}\{T C G\}\{T G C\}$



complementary

biases

(b)

Human colipase CLPS_1 (EPD database FP005405) $\quad-216 \rightarrow-105 \quad \mathrm{P}=5.505 \mathrm{e}-12 \quad$ \{AC

CGATG |CAACACAATTATCACCAACACACACACACACACACACACACACACACACACACACACGAAAAAGGCCACCAGGTACTCA

CCCAGTTTCCTTATCAGGAACCGCCCCACAGCA|GATTG

(c)

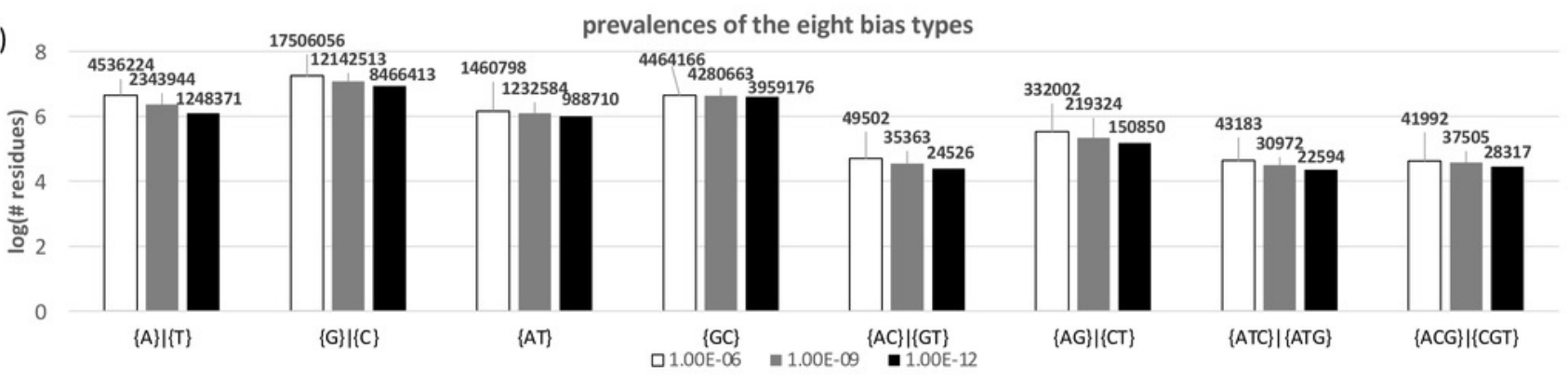




\section{Table $\mathbf{1}$ (on next page)}

Comparison of results for the precision options, using the yeast proteome as input 
1 Table 1: Comparison of results for the precision options, using the yeast proteome as input* 2

\begin{tabular}{|l|l|l|l|l|l|l|}
\hline & \multicolumn{2}{|l|}{ Number of single-residue CB regions } & \multicolumn{2}{l|}{ Number of multiple-residue CB regions } \\
\hline $\begin{array}{l}\text { P-value thresholds } \rightarrow \\
\begin{array}{c}\text { Precision option (-z) } \\
\downarrow\end{array}\end{array}$ & P $\leq 1 \mathrm{e}-03$ & P $\leq 1 \mathrm{e}-06$ & P $\leq 1 \mathrm{e}-09$ & $\mathrm{P} \leq 1 \mathrm{e}-03$ & $\mathrm{P} \leq 1 \mathrm{e}-06$ & $\mathrm{P} \leq 1 \mathrm{e}-09$ \\
\hline Fast (default) & 32022 & 5781 & 2268 & 6336 & 4512 & 2744 \\
\hline Medium & 36589 & 5792 & 2275 & 17117 & 6350 & 3395 \\
\hline Thorough & 37738 & 5792 & 2276 & 27229 & 7675 & 3766 \\
\hline
\end{tabular}

* UniProt reference proteome for S. cerevisiae 288C, downloaded January 2021. 


\section{Table 2 (on next page)}

Number of $S$. cerevisiae proteins with signal peptides that coincide with CB regions annotated by fLPS 
2 Table 2: Number of $S$. cerevisiae proteins with signal peptides that coincide with CB regions

3 annotated by fLPS*

\begin{tabular}{|c|c|c|c|}
\hline $\begin{array}{c}\text { P-value thresholds } \rightarrow \\
\text { Precision option (-z) } \\
\downarrow\end{array}$ & $P \leq 1 e-03$ & $P \leq 1 e-04$ & $P \leq 1 e-05$ \\
\hline Fast (default) & $60 / 301(19.9 \%)^{\star *}$ & $25 / 301(8.3 \%)$ & $15 / 301(5.0 \%)$ \\
\hline Medium & $111 / 301(36.9 \%)$ & $51 / 301(16.9 \%)$ & $29 / 301(9.6 \%)$ \\
\hline Thorough & $180 / 301(59.8 \%)$ & $90 / 301(29.9 \%)$ & 48/301 (15.9\%) \\
\hline
\end{tabular}

* Annotations for signal peptides were taken from UniProt (301 in total).

** The numbers of signal peptides for which $\geq 50 \%$ of their residues overlap $\geq 50 \%$ of the residues of an individual fLPS-

6 annotated CB region. 